\title{
Las tecnologías de la información y la comunicación como determinantes en el modelo de crecimiento de Corea del Sur: perspectivas hacia una alianza comercial con México
}

\author{
Information and Communication Technologies as \\ Determinants in the Growth Model of South Korea: \\ Perspectives towards a Commercial Alliance with Mexico
}

\section{DOI: $10.32870 /$ mycp.v10i28.702}

\section{Resumen}

A pesar de la ausencia de un acuerdo comercial bilateral entre Corea del Sur y México, una de las motivaciones que alienta esta formalización es el desarrollo acelerado que han tenido las tecnologías de la información y la comunicación (TIC) en el crecimiento económico coreano, pues han contribuido en la administración y organización de la industria, en la estandarización del comercio y la educación, entre otros rubros. El objetivo del estudio es analizar el impacto de las TIC como determinantes en el modelo de crecimiento económico de Corea del Sur dentro de un contexto regional que permita también detectar efectos particulares. Para ello se emplea un método de estimación de datos de panel durante el periodo 2000-2015. Los resultados indican que el modelo de crecimiento regional incorpora la formación bruta de capital fijo (FBCF) y la variable proxy de las TIC; adicionalmente se detecta un efecto positivo fijo para Corea del Sur.

Palabras clave: TIC, crecimiento económico, estimación de datos de panel, comercio exterior, tecnología.

\author{
Elsy Lizbeth Gómez Ramos ${ }^{1}$ \\ Carlos Gómez Chiñas ${ }^{2}$ \\ Daniel Jaime Camacho ${ }^{3}$
}

\begin{abstract}
Despite the absence of a bilateral trade agreement between South Korea and Mexico, one of the motivations that encourage this formalization from the Mexican government is the accelerated development that information and communication technologies (ICTs) have had in the economic growth in Korea, because they have contributed to the administration and organization of the industry, in the standardization of commerce and education, mainly. The objective of the study is to analyze the impact of ICTs as determinants in South Korea's economic growth model within a regional context and which in turn detect particular effects. For this, a panel data estimation method is used during the period 2000-2015. The results indicate that the regional growth model incorporates both gross fixed capital formation (GFCF) and the proxy variable for ICTs besides a positive effect for South Korea is detected.
\end{abstract}

Keywords: information technology, economic growth, panel data estimation, foreign trade.

Artículo recibido el 28 de mayo de 2020 y dictaminado el 24 de agosto de 2020.

1. Universidad Autónoma Metropolitana, Unidad Azcapotzalco, Departamento de Economía. Av. San Pablo 180, Col. Reynosa Tamaulipas, Alcaldía Azcapotzalco, C.P. 02200, Ciudad de México, México. ORCID: https://orcid.org/0000-0001-5670-4996 Correo electrónico: elsygomez@msn.com

2. Universidad Autónoma Metropolitana, Unidad Azcapotzalco, Departamento de Economía. Av. San Pablo 180, Col. Reynosa Tamaulipas, Alcaldía Azcapotzalco, C.P. 02200, Ciudad de México, México. ORCID: https://orcid.org/0000-0003-1162-7813 Correo electrónico: cgom@azc.uam.mx

3. Universidad Autónoma Metropolitana, Unidad Azcapotzalco, Departamento de Economía. Av. San Pablo 180, Col. Reynosa Tamaulipas, Alcaldía Azcapotzalco, C.P. 02200, Ciudad de México, México. ORCID: https://orcid.org/0000-0003-3367-3482 Correo electrónico: dnl.jaime@gmail.com 


\section{Introducción}

Las relaciones entre Corea del Sur y México pueden dimensionarse desde los ámbitos más generales hasta los más concretos. Entre los primeros destaca que de acuerdo con el Fondo Monetario Internacional (FMI), ambas economías se encuentran entre las 20 más importantes de mundo, ocupando para el año 2019 el puesto 12 (con un producto interno bruto [PIB] nominal de 1,629.53 mil millones de dólares) y 15 (con un PIB nominal de 1,274.18 mil millones de dólares), respectivamente. En ese mismo orden de ideas, estas naciones pertenecen a la Organización para la Cooperación y el Desarrollo Económico (OCDE) e integran el foro de discusión de líderes regionales MIKTA (por sus siglas en inglés de los países miembros) junto a Indonesia, Turquía y Australia (Secretaría de Relaciones Exteriores, 2015; Ortega \& Castañeda, 2016). Por otro lado, en ámbitos más concretos los dos países son parte de diversos acuerdos comerciales plurilaterales de alcance parcial (Organización Mundial del Comercio [OMC], 2020; Secretaría de Relaciones Exteriores, 2020) y del Grupo Conjunto de Expertos México-Corea del Sur (GCEMC), en los cuales se abordan temas sobre comercio y cooperación económica (De la Mora, 2012).

En consecuencia, un acuerdo comercial bilateral entre estas naciones ha generado grandes expectativas, sobre todo por la fuerza geopolítica de México (Ortega \& Castañeda, 2016), que lo posiciona como un enlace con la economía estadounidense. Una de las motivaciones que podría alentar esta formalización desde el Gobierno mexicano es el desarrollo acelerado que han tenido las tecnologías de la información y la comunicación (TIC) ${ }^{4}$ en el modelo de crecimiento económico coreano, toda vez que han contribuido en la administración y organización industrial, en la estandarización del comercio y la educación, entre otros rubros.

Lo anterior encuentra su fundamento teórico en el desarrollo del modelo de crecimiento de Solow, el cual establece que para lograr un crecimiento en el largo plazo es necesario el progreso tecnológico, pues la economía al entrar en el estado estacionario (debido a los rendimientos decrecientes) se funde en una condición de reposo, es decir, la tecnología (exógena) permite explicar cómo la función de producción puede continuar su trayectoria; de

4. De acuerdo con la Fundación del Español Urgente (FundéuRAE), la sigla adecuada para tecnologías de la información es TIC, con independencia de que una de las palabras de la expresión desarrollada aparezca en plural (Fundación del Español Urgente, 2014). 
hecho, se tiene evidencia de que la acumulación de capital en las economías desarrolladas se explica porque el progreso tecnológico permite neutralizar los rendimientos decrecientes, lo que implica un proceso de sustitución incesante (Easterly, 2001). Esta dinámica fue considerada por Schumpeter (1942) como un proceso de destrucción creativa que motiva la reconfiguración de la estructura de la industria. De este modo, Nelson y Winter (1982) conceptualizan a las invenciones e innovaciones como una combinación peculiar de recursos existentes, la cual es posible gracias a las interacciones existentes; por tanto, estas interacciones (entre las empresas e instituciones) son los impulsores de la innovación (Chandrashekar \& Bala Subrahmanya, 2019).

Entonces, ¿por qué no se ha concretado un acuerdo comercial bilateral con Corea del Sur? O, mejor dicho, ¿por qué las relaciones comerciales entre estas naciones siguen descansando principalmente bajo programas de fomento a la industria maquiladora de exportación? Algunas de las principales justificaciones de este letargo por parte de los responsables de la política exterior mexicana se amparan en la escasa vinculación de las empresas coreanas establecidas en nuestro país con la estructura productiva nacional, toda vez que sólo están encaminadas al uso intensivo de mano de obra barata (López, 2011a; Taddei \& Robles, 1997), mientras que otro argumento afín señala que las reducciones unilaterales de aranceles (en 2001, 2006 y 2009) a las importaciones por parte de México, provocó que Corea del Sur se sustente como el cuarto proveedor de nuestra economía, pero nunca significó una vinculación con las cadenas productivas ni tampoco un empuje real a las exportaciones mexicanas (De la Mora, 2012, pp. 85 y 86). En consecuencia, las actuales vinculaciones comerciales no han favorecido de forma importante al desarrollo de nuestro país, sino que sólo han tenido un efecto superficial que se caracteriza por un endeble encadenamiento a la estructura económica mexicana.

Una manera de empezar a resolver este conflicto (tener la oportunidad de negociar con Corea del Sur y la incapacidad de aprovechar esta fuente de conocimiento) es generar un acercamiento con el modelo de crecimiento coreano, pero dentro de un contexto regional que permita identificar efectos particulares. Para lograr este paso, se emplea un método de estimación de datos de panel, así resulta posible la oportunidad de generar un modelo y de resaltar efectos particulares. Sen y Saray (2019, p. 180) definen tres ventajas potenciales de estos modelos: 1) enfatizan los efectos de heterogeneidad; 2) permiten identificar otras relaciones, y 3 ) potencian los procesos de estimación. 
En general, las investigaciones que abordan las TIC para explicar el crecimiento económico medido como PIB bajo modelos de panel suelen identificarse por el tipo de economías analizadas. Por ejemplo, Mahmood y Bashir (2019) estudian un conjunto de países de ingresos bajos del sur de Asia (Pakistán, India, Bangladesh y Sri Lanka) para el periodo 1990-2014. Los resultados señalan que para este conjunto de países existe un proceso lento de absorción tecnológica. Así también, Sen y Saray (2019) estudian a la Unión Europea (28) y a Turquía como un conjunto de países desarrollados y subdesarrollados para el periodo 1997-2014. Las conclusiones señalan que tanto el capital físico como las TIC tienen una fuerte conexión para explicar el crecimiento económico. Y, finalmente, Afzal et al. (2019) incorporan tanto a las TIC como al PIB para explicar el consumo en electricidad para cinco economías emergentes: Brasil, Rusia, India, China y Sudáfrica (los BRICS) durante el periodo 19902014. Los resultados indican que al ser ambas variables significativas y con efectos positivos, existe una fuerte expansión de la infraestructura eléctrica, así como una transición hacia una sociedad digitalizada.

El objetivo del estudio es analizar el impacto de las TIC como determinante en el modelo de crecimiento económico (PIB) de Corea del Sur dentro de un contexto regional que permita identificar efectos particulares. Para ello se aplica un método de estimación de datos de panel durante el periodo 2000-2015.

Los resultados indican que el modelo de crecimiento regional se explica a través tanto de la formación bruta de capital fijo (FBCF) como de la variable proxy de las TIC (suscripciones en telefonía móvil); adicionalmente se detecta un efecto positivo para el caso de Corea del Sur. Lo anterior plantea a México las ventajas de la socialización de las TIC que podrían ayudar a cerrar brechas en diversos sectores bajo una planeación comercial dirigida.

El documento se divide en cuatro apartados, una conclusión y un apéndice. En el primero se presenta un marco de referencia sobre la apertura comercial de Latinoamérica orientada a Corea del Sur, principalmente. En el segundo se expone el funcionamiento de la herramienta seleccionada. En el tercero se muestra un análisis gráfico preliminar, la aplicación y los resultados del modelo. Y, finalmente, en el cuarto se presenta una discusión.

\section{Marco de referencia}

A partir de la firma del Tratado de Libre Comercio de América del Norte (TLCAN) en 1994, las relaciones comerciales para México se ampliaron mucho más 
Las tecnologías de la información y la comunicación como determinantes en el modelo de crecimiento de Corea del Sur: perspectivas hacia una alianza comercial con México

allá de los firmantes, pues significó el traslado de transnacionales japonesas y coreanas hacia el territorio mexicano, al ofrecer la posibilidad de abaratar costos y con ello consolidarse en la economía estadounidense. Para el caso de Japón, ${ }^{5}$ éste se posicionó con grandes oportunidades en el sector manufacturero (subsector automotriz y electrónico) al poder afianzar redes de insumos desde su lugar de origen y mantener una mínima inclusión con la economía mexicana (Falck, 2012). Bajo este panorama fue que en 2001 se iniciaron las negociaciones con Japón y en 2004 se firmó el Acuerdo de Asociación Económica México-Japón (AAEMJ) (Tratado de Asociación Transpacífico, 2018; Secretaría de Economía, 2020); no obstante, con el transcurso del tiempo la evolución del comercio evidencia un apego permanente a la triangulación comercial, ubicando a la economía estadounidense como el principal actor (Falck, 2012).

En este sentido, Japón representa para México no sólo la relación diplomática más longeva con la región, sino también la formalización de las relaciones comerciales a través del AAEMJ. Sin embargo, para León-Manríquez y Tzili (2015) esta relación comercial no obedece a la evolución diplomática que se materializó en la Comisión Mexicana de la Cuenca del Pacífico (CMCP) o en el Foro de Cooperación Económica Asia-Pacífico (APEC, por sus siglas en inglés), sino a las condiciones del mercado (Taddei \& Robles, 1997); de hecho, en los últimos 20 años la participación comercial japonesa se ha mantenido por debajo de lo que la inversión coreana representa.

En el caso de Corea del Sur la historia es más reciente, pues su desarrollo económico empezó en la década de los cincuenta, después de una guerra que terminó por dividir al país. Es sorprendente que en sólo medio siglo Corea del Sur haya transitado de ser uno de los países más pobres a ser una de las economías más desarrolladas del mundo, lo que también lo ha convertido en un atractivo socio comercial para Latinoamérica. De hecho, a principios del siglo XXI Chile se convirtió en el primer país de América Latina en firmar un acuerdo comercial con Corea del Sur en febrero de 2003 (aunque formalmente entró en vigor hasta 2004). La experiencia fue percibida como exitosa en Sud-

5. De acuerdo con Lal (2017), los flujos de la inversión extranjera directa (IED) no deben considerarse por sí solos como determinantes del crecimiento económico, toda vez que cada economía mantiene una naturaleza propia. Es decir, es posible que para ciertas economías la IED represente un impulso mínimo o casi nulo para el crecimiento económico. 
américa, de tal suerte que para los años 2010 y 2013 Corea del Sur firmaría nuevos acuerdos comerciales con Perú y Colombia, respectivamente.

De acuerdo con una publicación del Korean Knowledge Sharing Program y del Banco Interamericano de Desarrollo (2014), ${ }^{6}$ las relaciones entre Chile y Corea del Sur a partir de 2004 se han fortalecido. Estas conclusiones respecto al incremento de los flujos comerciales pudieron haber influido en la determinación de Perú y Colombia de concretar por su parte acuerdos comerciales con Corea del Sur; al menos así lo infieren Estevadeordal et al., (2015). Aun cuando esta hipótesis sea cierta, los resultados sí son distintos, pues en el caso de Perú si bien se nota un crecimiento de las exportaciones de aproximadamente el 100\% desde el primer año de vigencia del tratado, para Colombia sus exportaciones no parecen arrancar bien del todo, o al menos no con la fuerza que lo hicieron ni en Perú y mucho menos en Chile.

Por otro lado, las relaciones entre México y Corea del Sur se construyeron desde la década de los años sesenta del siglo pasado, década en la que Corea del Sur buscaba el reconocimiento internacional; de manera que se puede afirmar que su principal interés se encontraba en fomentar las relaciones políticas y diplomáticas, antes que las económicas y comerciales (López, 2011b); sin embargo, una vez alcanzado el primero, claramente ha llevado sus objetivos al siguiente nivel. Las operaciones comerciales entre ambas economías han sido crecientes, tan sólo a principios del siglo actual, durante el año 2000, el volumen de comercio exterior apenas alcanzaba los $\$ 3,984 \mathrm{mdd}$, pero para el cierre de 2019 el volumen total fue del orden de $\$ 19,789 \mathrm{mdd}$, lo que nos dice que el comercio entre ambos países se ha quintuplicado en tan sólo 20 años (un crecimiento de $483 \%$ ). ${ }^{7}$ Este desempeño coloca a Corea del Sur como tercer socio comercial de México en el continente asiático y de manera global en el quinto proveedor. Si bien este dato es marginal en un país como México, con alta concentración de su comercio exterior y sólo representa un 2\%-3\% del total importado nacional, se deja entrever la tenacidad de los coreanos y

6. La misma opinión sobre la importancia que ha adquirido la república de Corea de Sur para América Latina la comparte la plataforma ConnectAmericas, que es una plataforma de negocios creada por el BID y un conjunto de empresas cuyo objetivo es expandir las oportunidades en la región y bajar la incidencia de fracasos en las que llegan a caer las pymes cuando buscan expandirse (ConnectAmericas, s.f. https://connectamericas.com/es/content/conoce-cu\%C3\%A1les-son-lostlc-vigentes-entre-corea-del-sur-y-lac

7. Cifras estimadas con información de la United Nations Comtrade Database y con base en el Sistema de Clasificación, Designación y Codificación de Mercancías del Sistema Armonizado (United Nations, 2020). 
hace que se retome el debate sobre la condición deseable de la diversificación para la autonomía política de los Estados (Montes \& López, 2018).

El interés de concretar un tratado de libre comercio con México por parte de Corea del Sur puede entenderse distinto al interés que prevalecía con países sudamericanos, en el sentido de que México se encuentra en una posición geográfica ventajosa, pues no hay que dejar de observar el hecho de que nuestro país es el vecino latinoamericano más cercano a Estados Unidos y cuenta con un acuerdo que le confiere ciertas facilidades en el comercio intraindustrial e intrafirma. Este hecho explica, de acuerdo con López (2011b), que aproximadamente el $70 \%$ del comercio bilateral entre ambos países sea de manufacturas; de manera más específica, de insumos y bienes intermedios que se incorporan a bienes finales y se re-exportan a Estados Unidos desde México.

El comercio internacional de manufacturas, a diferencia de los bienes primarios, podría llevar implícita la transferencia de conocimiento y tecnología y, por lo tanto, cierto nivel de capacidades tecnológicas. En esta misma idea, pero desde una perspectiva más ligada a la empresa y al producto, Dosi y Nelson (2010) señalan que la innovación es un proceso evolutivo compuesto por dinámicas industriales (empresariales o sectoriales), crecimiento económico (variables macroeconómicas) y la propia innovación tecnológica y organizativa.

El impacto que la tecnología y las actividades de investigación y desarrollo tienen en variables relacionadas con el crecimiento económico han sido ligadas a la productividad y a la profundización del capital tangible e intangible (David \& Foray, 2002), por lo que su tratamiento a través de la metodología econométrica es un aspecto importante en la búsqueda de la estimación de los impactos que las variables tienen al respecto.

\section{Modelo panel}

De acuerdo con Pérez (2008, pp. 271-290), la particularidad de un conjunto de datos panel es la combinación entre una serie temporal y una dimensión de sección cruzada. La primera, definida como la secuencia de valores que toma una variable en el tiempo para una unidad de análisis; por tanto, una serie temporal $X$ incluye la secuencia de valores $X_{t-i} \operatorname{con} i=1,2,3, \ldots, T$, siendo $T$ el número de momentos en el tiempo para cada unidad social de análisis. Y la segunda, definida como el estudio de un conjunto de unidades sociales durante un determinado tiempo; aquí cada observación incluye valores para 
ciertas variables que caracterizan a cada una de las unidades sociales; por tanto, una sección cruzada los valores para variables $X_{k}$, donde $k=1,2,3, \ldots$ $K$, siendo $K$ el número de variables para identificar la unidad social. Las clases de modelos más empleados son: los modelos de coeficientes constantes, los modelos de efectos fijos y los modelos de efectos aleatorios.

En el modelo de coeficientes constantes se asume que los coeficientes son los mismos para cada uno de los agentes sociales, se tiene que:

$$
y_{i t}=\beta_{0}+\sum_{k=1}^{k} \beta_{k} x_{k i t}+u_{i t}
$$

Donde $k=1, \ldots K$ variables independientes, $i=1, \ldots, N$ unidades sociales y $t=1, \ldots, T$ observaciones en el tiempo. Los $K$ parámetros se consideran iguales o constantes para todas las unidades de las muestras, así como para cada periodo de tiempo. La estimación de mínimos cuadrados ordinarios (MCO) parte del supuesto de que la varianza de los términos de error es la misma para cada una de las observaciones (supuesto de homocedasticidad) y que estos no estén correlacionados. Adicionalmente, se puede asumir que existe correlación serial, donde para cada unidad social los términos de errores están correlacionados a través del tiempo. Pero si se incorpora un autorregresivo, en general, de primer orden es posible corregir el problema.

En el caso de un modelo de efectos fijos, se tiene como supuesto que los coeficientes (la constante o término independiente del modelo de regresión) varían dependiendo del agente social (que pueden ser individuos, empresas o países) o del momento en el tiempo. Cuando el componente transversal es el dominante, el modelo puede captar la variación existente en la muestra debido a la presencia de diferentes agentes sociales con la inclusión de variables dicotómicas $d_{i}$ cuyos coeficientes asociados en el modelo de regresión son $a_{i}$ (una variable para cada agente social, menos el agente social de referencia). El modelo de regresión a estimar es:

$$
y_{i t}=\beta_{0}+a_{1} d_{1}+a_{2} d_{2}+\cdots+a_{N} d_{N}+\sum_{k=1}^{k} \beta_{k} x_{k i t}+u_{i t}+\varepsilon_{i t}
$$

En este modelo se permite que los efectos individuales $a_{i}$ puedan estar correlacionados con las variables explicativas, pero para que los estimadores por MCO sean consistentes se requiere la exogeneidad estricta de $X_{i t}$ y $\varepsilon_{i t}$.

En el modelo de coeficientes aleatorios se asume que los coeficientes individuales $a^{i}$, no son efectos fijos en el término independiente de la regresión, 
sino que estos pueden variar de manera aleatoria en el tiempo a través de los agentes sociales. Aunque existen varias versiones de este modelo, la más utilizada es el modelo con varios componentes de error. Éste emplea un error aleatorio en el tiempo, un error aleatorio en las unidades sociales, y un error que depende del tiempo y de las unidades sociales pero que es aleatorio; con ello se buscan estimaciones eficientes y no sesgadas de los coeficientes de la regresión. Para el caso más general, este modelo de regresión es el siguiente:

$$
y_{i t}=\beta_{0}+\sum_{k=1}^{k} \beta_{k} x_{k i t}+u_{i t}
$$

Donde $y_{i t}$, es una función lineal de $K$ variables explicativas, y el término de error tiene la siguiente estructura:

$$
u_{i t}=a_{i}+\phi_{t}+\varepsilon_{i t}
$$

Donde $i=1, \ldots, N$ unidades sociales y $t=1, \ldots, T$ observaciones en el tiempo.

El error $u_{i t}$ tiene un componente individual aleatorio que es invariable a $t$ ravés del tiempo $a_{i t}$ (componentes "entre grupos") y un componente temporal aleatorio que es invariable a través de los individuos $\phi_{t}$ (componente "intragrupos") y $\varepsilon_{i t}$ el componente aleatorio. Para cada uno de estos componentes del error total, se sigue con los supuestos del modelo de regresión lineal.

\section{Aplicación}

Análisis gráfico preliminar

Bajo el entorno de los países miembros de APEC, se analiza el impacto de las TIC en el crecimiento económico para el año 2015.

La figura 1 muestra la relación del índice de desarrollo TIC y el PIB per cápita (a precios constantes en dólares) para los miembros del APEC durante 2015. Los resultados señalan que existe una relación positiva entre los datos; de hecho, al trazar una función lineal el comportamiento se hace legible. Para el caso de México, se mantiene un índice de desarrollo Tic bajo (4.50) al igual que un nivel del PIB per cápita ( $\$ 9,650.95$ dólares), mientras que en el extremo derecho de la figura se encuentra Corea del Sur (República de Corea, nombre oficial) con el mayor índice de desarrollo TIC (8.80) —no sólo para la muestra seleccionada, sino también a nivel mundial—y con un nivel del PIB per cápita 
(\$27,105.076 dólares) que se ubica por arriba de la media. En esencia, las TIC ayudan a explicar de manera importante el nivel de riqueza de una nación.

Muestra

La muestra incluye a los países asiáticos ${ }^{8}$ (China, Hong Kong, Japón, Malasia, Mongolia, República de Corea, Rusia, Tailandia y Singapur) que aparecen en la figura 1.

8. No se considera a Vietnam debido a la falta de datos durante el periodo analizado.

80 México y la Cuenca del Pacífico. Vol. 10, núm. 28 / enero-abril de 2021. 
Las tecnologías de la información y la comunicación como determinantes en el modelo de crecimiento de Corea del Sur: perspectivas hacia una alianza comercial con México

\section{Figura 1}

Países miembros de APEC: relación entre el índice de desarrollo TIC y el PIB per cápita (2015)

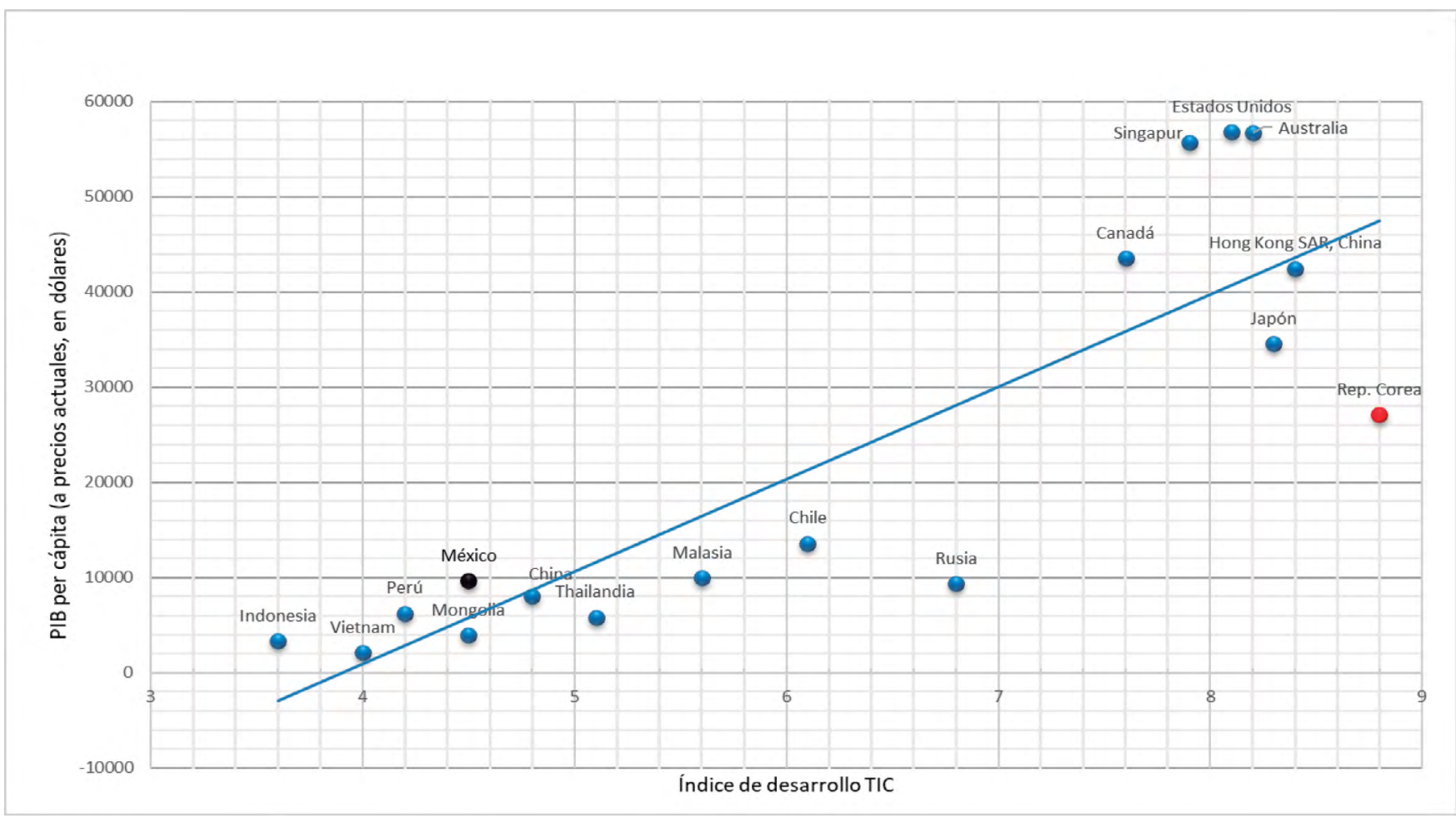

Fuente: elaboración propia con base en los datos del Banco Mundial (2020a, 2020b).

México y la Cuenca del Pacífico. Vol. 10, núm. 28/enero-abril de 2021. Análisis 81 
Modelo

En términos de las especificaciones del modelo, se consideran los planteamientos de Sen y Saray (2019) y de Mahmood y Bashir (2019), es decir, donde el crecimiento de una economía no sólo se compone de capital físico, sino también de la tecnología desde el ámbito de las Tic, ${ }^{9}$ quedando como sigue:

$P I B_{i t}=$ Telefonía Móvilit $+F B C F_{i t}+\varepsilon_{i t}$

Donde $P I B_{i t}$, es el logaritmo natural del PIB per cápita del país $i$ al tiempo $t$, TelefoníaMóvilit, es el logaritmo natural de suscripciones en telefonía móvil (por cada 100 personas) y $F B C F_{i t}$, es el logaritmo natural de la formación bruta de capital fijo (porcentaje del PIB). La frecuencia de los datos es anual durante el periodo 2000-2015. La fuente de los datos es el Banco Mundial (2020a). El software utilizado es EViews 9.

En relación con el apartado anterior, se tiene que $N=9, K=2$ y $T=16$, es decir, nueve países (China, Hong Kong, Japón, Malasia, Mongolia, República de Corea, Rusia, Tailandia y Singapur), dos unidades sociales (Telefonía_Móvil y FBCF) y 16 observaciones por país para cada unidad social.

Los resultados del modelo se resumen en la tabla 1, la cual se divide en dos partes: pruebas básicas y las regresiones. La primera parte muestra los resultados de las pruebas de heteroscedasticidad y de autocorrelación, lo que permite aceptar la igualdad de las varianzas residuales y la corrección de la autocorrelación al incorporar un autorregresivo de primer orden (AR [1]). La segunda parte especifica la regresión simple por MCO sin efectos, la cual muestra buena significancia individual (Telefonía_Móvil y FBCF) y global (prob. F), y con un nivel del estadístico Durbin Watson superior al coeficiente de determinación — cumpliendo la regla general—, mientras que el modelo de efectos fijos ${ }^{10}$ indica un nivel de significancia individual y un ajuste del modelo bien posicionados, aunque el error estándar de la regresión (ee) fue

9. Se analizaron diversas variables como proxy a las TIC (suscripciones en banda ancha, suscripciones a telefonía fija, etc.) para la región; no obstante, de acuerdo con el análisis preliminar ninguna variable mantenía una relación positiva con el PIB durante el periodo seleccionado, sólo las suscripciones en la telefonía móvil.

10. Véase el Apéndice, para el análisis gráfico individual de las series y la visualización de la salida del software del modelo sin efectos. 
mayor y, finalmente, la prueba Hausman muestra que el modelo de efectos aleatorios no es un modelo apropiado.

La ecuación del modelo de efectos fijos es la siguiente:

$$
\begin{aligned}
& P I B_{i t}=4.625593+0.593612 \text { TelefoníaMóvilit }+0.605068 F B C F_{i t}-0.2858 d_{1} \\
& +1.0896 d_{2}-0.8918 d_{3}-1.0671 d_{4}+0.5166 d_{5}+0.8223 d_{6}+1.3754 d_{7} \\
& -1.4830 d_{8}-0.0762 d_{9}+\varepsilon_{i t}
\end{aligned}
$$




\section{Tabla 1}

Estimaciones para la variable dependiente PIB per cápita

Pruebas básicas $^{\mathrm{a}}$

Heteroscedasticidad

Pruebas de igualdad de varianza residual

Método

Barlett (prob. $=0.3591)$

Levene (prob. $=0.1884)$

Brown-Forsythe (prob. $=0.3045$ )

Autocorrelación

Durbin Watson $=0.133078$ Con $\operatorname{Ar}(1)$ DW $=1.604018$

\begin{tabular}{l|l|l|l}
\hline & c & Telefonía_Móvil & fbc_f \\
\hline $\begin{array}{l}\text { MCO } \\
R^{2}=0.99243, e e=0.10585, \mathrm{AR}(1)=0.96611\end{array}$ & $(0.0000)$, Prob. (F-estad.) $=0.000$ \\
\hline Coeficiente & 9.270508 & 0.083105 & 0.432982 \\
\hline Error estándar & 0.615956 & 0.034344 & 0.114369 \\
\hline Probabilidad & $(0.0000)^{\mathrm{b}}$ & $(0.0169)$ & $(0.0002)$ \\
\hline $\begin{array}{l}\text { Modelo de efectos fijos } \\
R^{2}=0.970260, \text { ee }=0.22104\end{array}$ & & \\
\hline Coeficiente & 4.625593 & 0.593612 & 0.605068 \\
\hline Error estándar & 0.566869 & 0.028740 & 0.186146 \\
\hline Probabilidad & $(0.0000)$ & $(0.0000)$ & $(0.0015)$ \\
\hline
\end{tabular}

Modelo de efectos aleatorios

Prueba Hausman (0.0026)

\begin{tabular}{l|l|l|l}
\hline Coeficiente & 4.814444 & 0.602720 & 0.535454 \\
\hline Error estándar & 0.602065 & 0.028559 & 0.183162 \\
\hline Probabilidad & $(0.0000)$ & $(0.0000)$ & $(0.0040)$ \\
\hline
\end{tabular}

${ }^{a}$ En términos de la heteroscedasticidad, se encuentra evidencia de que no existe entre las secciones cruzadas (p-valor es mayor a 0.05). Para el caso de la autocorrelación, se intenta corregir a través de un autorregresivo de primer orden. De acuerdo con las tablas, el valor inferior de DL es 1.598 y DU es 1.651, por lo tanto, el valor DW $=1.604018$ cae en la zona de indecisión.

${ }^{\mathrm{b}}$ Entre paréntesis se indican los coeficientes significativos al $5 \%$.

c La prueba de Hausman señala que el modelo de efectos aleatorios no es apropiado.

Fuente: elaboración propia.

Donde $d_{i}$, indica los efectos fijos de las secciones cruzadas. Con ello se expresa una diferenciación individual en los niveles del crecimiento económico que se atribuyen a los efectos propios de cada país (tabla 2). 
Tabla 2

Efectos fijos por país

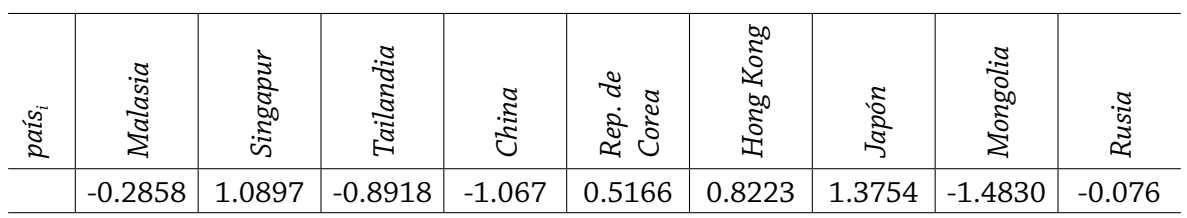

Fuente: elaboración propia con base en la ecuación 2.

Los resultados indican que el modelo de crecimiento regional incorpora tanto la formación bruta de capital fijo (FBCF) como la variable proxy de las TIC (suscripciones en telefonía móvil por cada 100 personas); adicionalmente se detecta un efecto fijo positivo para Corea del Sur. Lo anterior sugiere que la influencia regional prevalece en el tiempo y que existe una relación interna para Corea del Sur que impulsa su crecimiento económico. Respecto a la relación interna positiva (o efecto fijo) detectada en el modelo, no conocemos su naturaleza, pero se puede inferir que el nivel educativo, otras tecnologías TIC como la banda ancha (este país cuenta con la banda ancha más sofisticada a nivel mundial) o las interrelaciones entre estos (capital humano y tecnologías TIC) y otros puedan tener una relevancia importante.

\section{Discusión}

El anterior apartado deja a la reflexión que el "milagro coreano" obedece a elementos multifactoriales e incluso algunos de ellos difíciles de definir de manera exacta. Por ejemplo, la estructura del modelo educativo de Corea del Sur está fuertemente vinculada a la participación del Estado que procuró el monitoreo, la estandarización y la cobertura de la educación en todos los niveles (Martínez, 2007). Para el caso de la educación superior, Licona y Rangel (2013) concluyen que debido a la relación estratégica entre Estado y mercado, se ha podido absorber adecuadamente a esta fuerza laboral calificada, toda vez que se encuentra articulada la educación con las prioridades nacionales, entre ellas la innovación.

En este sentido, para México los retos más evidentes surgen en el mosaico multicultural y en la dispersión de la población, así como en las concesiones otorgadas a la iniciativa privada (Martínez, 2007). En consecuencia, en au- 
sencia de un plan nacional de mejora educativa en todos los niveles no será posible una adecuada inserción al mundo actual, pues el rezago educativo tenderá a generar mayores brechas.

El ejemplo anterior concuerda con lo analizado por De la Mora (2012, p. 20), que señala al sector privado como el principal promotor de la suspensión de las negociaciones con Corea del Sur, pues eso significaría una mayor competencia en las telecomunicaciones, transporte, energía y educación.

Lo anterior no significa que la apertura comercial se deba tomar sin un marco regulatorio pertinente, sino más bien que las políticas públicas en México no están articuladas adecuadamente para lograr un plan nacional de desarrollo económico efectivo.

Lo que se propone en esta investigación es tomar ventaja de lo que nos puede ofrecer Corea del Sur: conocimiento. Este conocimiento se puede ramificar desde la adopción de las Tic para la educación, hasta el diseño de centros de investigación binacionales.

De la Mora (2012, pp. 155 y 156) ha expuesto iniciativas previas a una apertura comercial con Corea del Sur enfocadas en la iniciativa privada: a) acceso al crédito; b) liberar el sector servicios; c) impulsar la infraestructura y la logística; d) desarrollar una política industrial basada en la innovación, y e) internacionalizar las pymes. No obstante, en esta investigación se aboga por la iniciativa de la liberación del sector servicios (inciso b) a partir de acciones incluyentes para toda la población (en la educación, pero también en la seguridad y en la salud; en las cuales es muy probable alcanzar resultados sorprendentes con las TIC), previo a lo dicho por De la Mora (2012). Es decir, si el modelo desarrollado aquí especifica una relación positiva entre el PIB y las suscripciones en telefonía móvil, entonces se muestra evidencia de que la socialización de la tecnología ayuda en el crecimiento. Pero si se liberan los mercados sin que exista, en general, una población capaz de aprovechar estos beneficios, entonces se dará una mayor concentración del conocimiento.

\section{Conclusiones}

La consolidación de las relaciones comerciales y financieras de México con Corea del Sur en los pasados cincuenta años se ha registrado en un entorno en el que la falta de un tratado comercial no parece haber sido un obstáculo; no debería de serlo ahora tampoco. Si bien es cierto que esa relación se muestra marginal y poco significativa, la experiencia con otros tratados no 
garantiza que se transforme drásticamente y se convierta en un verdadero polo de diversificación; sin embargo, lo que sí puede decirse es que la relación México-Corea del Sur sigue mostrando grandes áreas de oportunidad.

Para el grupo de países analizados (China, Hong Kong, Japón, Malasia, Mongolia, República de Corea, Rusia, Tailandia y Singapur) durante el periodo de estudio (2000-2015), el crecimiento económico se puede explicar a través de las suscripciones en telefonía móvil (por cada 100 personas) y de la formación bruta de capital fijo, es decir, por una variable análoga a la tecnología y por el capital físico. Los resultados señalan que se obtiene un efecto fijo positivo para Corea del Sur, posicionándose después de Japón, Singapur y Hong Kong.

Lo anterior permite obtener evidencia de que la dinámica en el crecimiento económico de Corea del Sur también refiere a elementos muy particulares, el más conocido es la estructura educativa. Por lo tanto, se concluye que el manejo de las TIC podría orientarse en primer lugar a subsanar problemáticas que aquejan a México (educación, seguridad y salud), y posteriormente buscar potenciar los beneficios a largo plazo de una alianza comercial.

Finalmente, en caso de que se concretara de manera efectiva un acuerdo de carácter comercial y financiero con Corea del Sur, éste debería privilegiar que el interés se centre en el escalamiento tecnológico de la oferta exportable desde Corea del Sur y hacia cualquier otro país, lo que significa la generación de mayor valor agregado, es decir que se vaya más allá de la mera reducción arancelaria de los bienes y servicios motivo del comercio bilateral. En nuestra opinión, las firmas de los tratados de libre comercio deben ser acompañadas por una visión de largo plazo que fomenten el crecimiento mutuo y que tiendan a reducir las condiciones de desigualdad de las economías participantes en todos los ámbitos, sean comerciales, financieros, tecnológicos, medioambientales, etc., y que no se basen casi de manera exclusiva en las oportunidades que la complementariedad y las ventajas comparativas ofrecen. 


\section{Apéndice. Comportamiento de las variables seleccionadas para el modelo panel}

Figura $1 a$

Logaritmo natural del PIB per cápita (2000-2015)

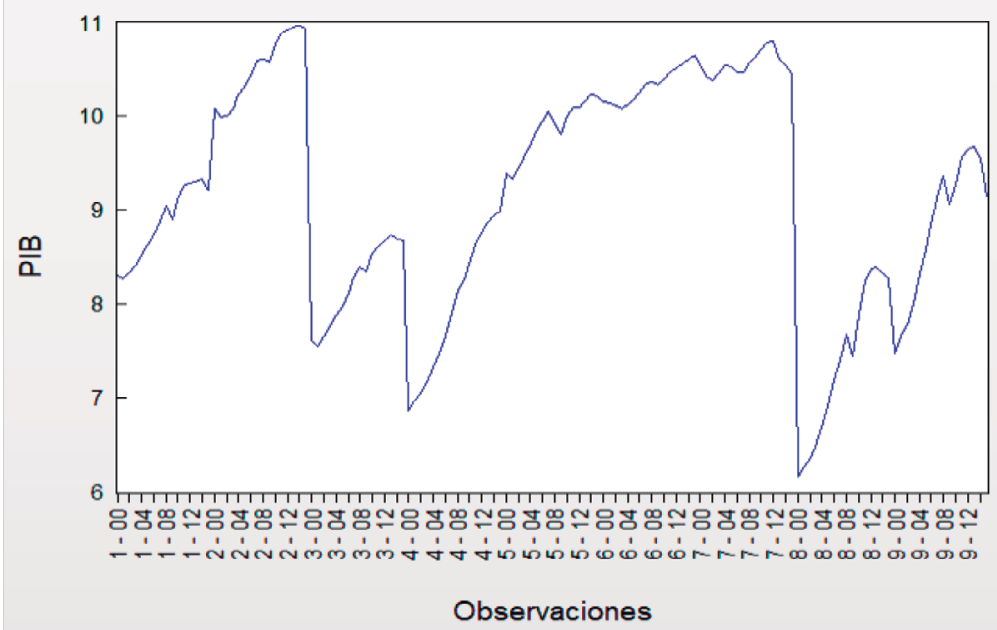

Figura $1 b$

Logaritmo natural de la formación bruta de capital fijo (2000-2015)

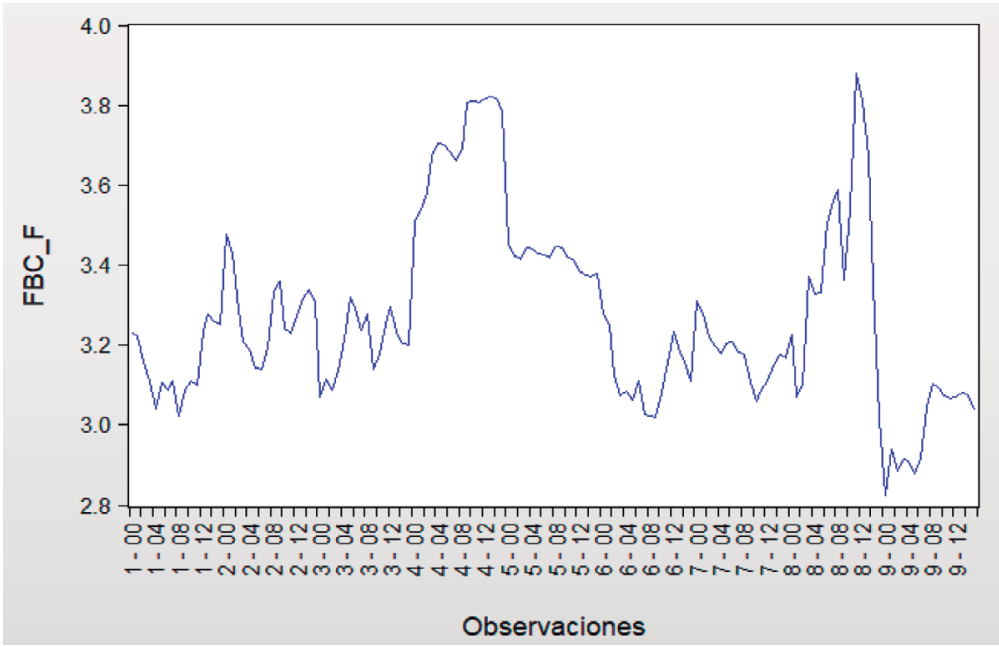


Las tecnologías de la información y la comunicación como determinantes en el modelo de crecimiento de Corea del Sur: perspectivas hacia una alianza comercial con México

Figura 1c

Logaritmo natural de suscripciones en telefonía móvil (2000-2015)

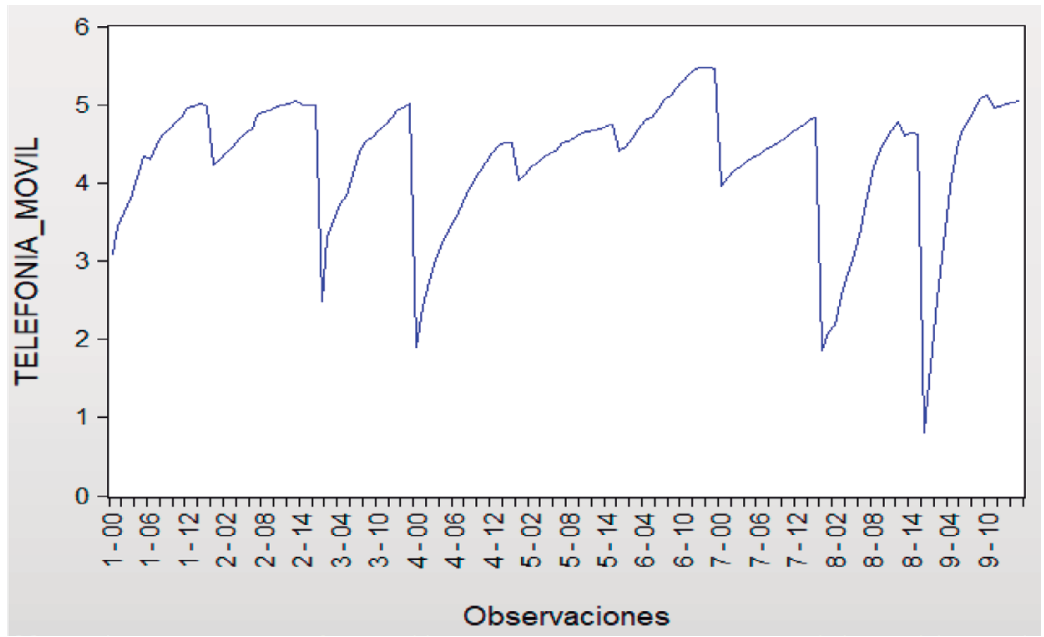

Figura $2 a$

Modelo sin efectos en EViews

Dependent Variable: PIB

Method: Panel Least Squares

Date: $07 / 07 / 20$ Time: $14: 34$

Sample (adjusted): 20012015

Periods included: 15

Cross-sections included: 9

Total panel (balanced) observations: 135

Convergence achieved after 6 iterations

\begin{tabular}{cclll}
\hline \hline \multicolumn{1}{c}{ Variable } & Coefficient & Std. Error & t-Statistic & Prob. \\
\hline \hline C & 9.270508 & 0.615956 & 15.05060 & 0.0000 \\
TELEFONIA_MOVIL & 0.083105 & 0.034344 & 2.419764 & 0.0169 \\
FBC_F & 0.432982 & 0.114369 & 3.785824 & 0.0002 \\
AR(1) & 0.966119 & 0.007630 & 126.6283 & 0.0000 \\
\hline \hline R-squared & 0.992439 & Mean dependent var & 9.207593 \\
Adjusted R-squared & 0.992266 & S.D. dependent var & 1.203645 \\
S.E. of regression & 0.105852 & Akaike info criterion & -1.624375 \\
Sum squared resid & 1.467798 & Schwarz criterion & -1.538293 \\
Log likelihood & 113.6453 & Hannan-Quinn criter. & -1.589394 \\
F-statistic & 5731.780 & Durbin-Watson stat & 1.604018 \\
Prob(F-statistic) & 0.000000 & & & \\
\hline \hline Inverted AR Roots & .97 & & & \\
\hline \hline
\end{tabular}




\section{Referencias}

Afzal, M. N. I., Gow, J., \& Rahman, A. (2019). Economic and Internet Growth Effect on Electricity Consumption in the BRICS Countries. International Advances in Economic Research, 25, 339-346. https://doi.org/10.1007/ s11294-019-09743-6

Banco Mundial. (2020a). Indicadores del desarrollo mundial. https://databank. bancomundial.org/id/8108d90c?Report_Name=World-DevelopmentIndicators\#

Banco Mundial. (2020b). Country Rank and Value in the ICT Development Index. Indicador, TCdata360. https://tcdata360.worldbank.org/indicators/ h2e1ddd20 ? country=BRA\&indicator $=24719 \& v i z=$ bar_chart\&year$\mathrm{s}=2015$ \&indicators $=944$

Chandrashekar, D., \& Bala Subrahmanya, M. (2019). Exploring the factors of cluster linkages that influence innovation performance of firms in a cluster. Economics of Innovation and New Technology, 28(1), 1-22. https:// doi.org/10.1080/10438599.2017.1384102

ConnectAmericas. (s.f.). Conoce cuáles son los TLC vigentes entre Corea del Sur yLAC. https://connectamericas.com/es/content/conoce-cu\%C3\%A1lesson-los-tlc-vigentes-entre-corea-del-sur-y-lac

David, P., \& Foray, D. (2002). An introduction to the economy of the knowledge society. International Social Sciences Journal, 54(171), 9-23. https://doi. org/10.1111/1468-2451.00355

De la Mora, L. M. (2012). Apertura con reciprocidad. Cómo reinsertar a México en la economía global. Centro de Investigaciones y Docencia Económicas.

Dosi, G., \& Nelson, R. (2010). Technical Change and Industrial Dynamics as Evolutionary Processes. En B. H. Hall y N. Rosenberg (Eds.), Handbook of the Economics of Innovation, (Vol. 1, pp. 51-127). Elsevier. https://doi. org/10.1016/S0169-7218(10)01003-8

Easterly, W. (2001). En busca del crecimiento. Andanzas y tribulaciones de los economistas del desarrollo. Antoni Bosch.

Estevadeordal, A., Mesquita M. M., \& Kahn T. (2015). Korea and Latin America and the Caribbean: Striving for a diverse and dynamic relationship. InterAmerican Development Bank. https://publications.iadb.org/publications/ english/document/Korea-and-Latin-America-and-the-Caribbean-Striving-for-a-Diverse-and-Dynamic-Relationship.pdf

Falck, M. (2012). Japanese Foreign Direct Investment in Mexico and the Impact of the Global Crisis. Revista Globalización, Competitividad y Gobernabilidad, 6(1), 36-54. https://doi.org/10.3232/GCG.2012.V6.N1.03 
Fundación del Español Urgente (FundéuRAE). (2014, abril 24). las TIC, major que las TICs o las TICS. https://www.fundeu.es/recomendacion/las-ticmejor-que-las-tics-o-las-tics/

Korean Knowledge Sharing Program \& Inter-American Development Bank (IDB). (2014). Joint Consulting with IOs: Promotion of SMEs in LAC through Connect Americas. https://www.kdevelopedia.org/resource/ view/04201611110146567.do\#.X06zZ4uZKUk

Lal, A. K. (2017). Foreign Direct Investment, Trade Openness and GDP in China, India, and Mexico. The Singapore Economic Review, 62(5), 10591076. https://doi.org/10.1142/S0217590815501076

León-Manríquez, J. L., \& Tzili, E. (2015). México y Asia Pacífico: proximidades y distancias de una dilatada relación. Revista CIDOB d'Afers Internacionals, (110), 113-139. https://www.cidob.org/es/articulos/revista_cidob_d_ afers_internacionals/110/mexico_y_asia_pacifico_proximidades_y_distancias_de_una_dilatada_relacion

Licona, A., \& Rangel, J. E. (2013). Pilares de la competitividad, educación superior, nuevas tecnologías y empleo en Corea del Sur y México. Análisis Económico, 69 (28), tercer cuatrimestre, 79-108.

López, J. F. (2011a). Comercio e inversión coreana en México: el TLC bilateral como víctima de respuestas divergentes a la crisis. México y la Cuenca del Pacífico, 14(41), 31-64. https://doi.org/10.32870/mycp.v14i41.366

López, J. F. (2011b, octubre 25 y 26). La vinculación de Corea con América Latina y México tras 50 años de relaciones diplomáticas: realidades y desafíos [Ponencia]. XII Seminario Internacional de Estudios sobre la Cuenca del Pacífico, Universidad de Colima, Colima, México. http://www.portesasiapacifico. com.mx/CUEICP/memorias/Sem12/memorias.php?ponencia $=190$

Mahmood, T., \& Bashir, S. (2019). Measuring the Impact of Information and Communication Technology Capital (ICT-Capital) on Economic Growth: Evidence from SAARC Countries. Pakistan Business Review, 21(1), 162-175. https://pbr.iobm.edu.pk/wp-content/uploads/2019/10/14-Article-1.pdf Martínez, F. (2007). La educación en México y en Corea del Sur; Cuaderno 27 Colección Cuadernos de Investigación. INEE. https://www.inee.edu.mx/ wp-content/uploads/2019/01/P1C135.pdf

Montes, E. Y., \& López, J. F. (2018). Corea del Sur como opción para la diversificación de relaciones económicas de México. Chakana. Revista Internacional de Estudios Coreanos, 2, 157-182.

http://www.catedrasinternacionales.ucr.ac.cr/chakana/2018/12/28/montesy-lopez/ 
Nelson, R., \& Winter, S. (1982). An Evolutionary Theory of Economic Change. The Belknap Press of Harvard University Press.

Organización Mundial del Comercio (OMC). (2020). Regional Trade Agreements, database. http://rtais.wto.org/UI/PublicMaintainRTAHome.aspx

Ortega, H., \& Castañeda, L. A. (2016). MIKTA: ampliar los alcances de la política exterior mexicana. Revista Mexicana de Política Exterior, (108), 211-228.

https://revistadigital.sre.gob.mx/images/stories/numeros/n108/ortegacastaneda.pdf

Pérez, C. (2008). Econometría avanzada. Técnicas y herramientas. Pearson.

Schumpeter, J. A. (1942). Capitalismo, socialismo y democracia. Orbis.

Secretaría de Economía. (2020, abril 01). El Acuerdo de Asociación Económica México-Japón celebra su 15 aniversario. https://www.gob.mx/se/ articulos/el-acuerdo-de-asociacion-economica-mexico-japon-celebra-su15-aniversario?idiom $=\mathrm{es}$

Secretaría de Relaciones Exteriores. (2015, marzo 18). ¿Qué es el MIKTA y cuál es el papel de \#MéxicoGlobal? https://www.gob.mx/sre/articulos/que-esmikta-y-cual-es-el-papel-de-mexicoglobal

Secretaría de Relaciones Exteriores. (2020). Acuerdo sobre el sistema global de preferencias comerciales entre países en desarrollo.

https://aplicaciones.sre.gob.mx/tratados/ARCHIVOS/PREFERENCIASPAISES\%20EN\%20DESARROLLO.pdf

Sen, A., \& Saray, M. (2019). Information and Communication Technologies and Economic Growth: Evidence from EU and Turkey. Journal of Economic Cooperation and Development, 40(2), 169-192. https://jecd.sesric.org/pdf.php?file=ART18051701-2.pdf

Taddei, C., \& Robles, J. (1997). Estrategias de expansión de las firmas japonesas y coreanas hacia la frontera norte de México. Problemas del Desarrollo. Revista Latinoamericana de Economía, 28(111), 123-148. https://doi.org/10.22201/iiec.20078951e.1997.111.28413

Tratado de Asociación Transpacífico. (2018, diciembre 30). El TIPAT entra en vigor y abre nuevos mercados para México. https://www.gob.mx/tratado-deasociacion-transpacifico/articulos/el-tipat-entra-en-vigor-y-abre-nuevosmercados-para-mexico-186182

United Nations. (2020). UN Comtrade Database. https://comtrade.un.org/ data/ 